\section{Cahiers de Narratologie}

Analyse et théorie narratives

8 | 1997

Création de l'espace et narration littéraire

\title{
Espaces romanesques, espaces du souvenir : le chemin d'une écriture
}

\section{Montserrat Mañes Serrano}

\section{OpenEdition}

1 Journals

Édition électronique

URL : https://journals.openedition.org/narratologie/11658

DOI : 10.4000/narratologie.11658

ISSN : 1765-307X

Éditeur

LIRCES

\section{Édition imprimée}

Date de publication : 1 décembre 1997

Pagination : 343-356

ISBN : 291089746X

ISSN : 0993-8516

Référence électronique

Montserrat Mañes Serrano, «Espaces romanesques, espaces du souvenir : le chemin d'une écriture », Cahiers de Narratologie [En ligne], 8 | 1997, mis en ligne le 15 décembre 2020, consulté le 21 septembre 2021. URL : http://journals.openedition.org/narratologie/11658; DOI : https://doi.org/10.4000/ narratologie. 11658

Ce document a été généré automatiquement le 21 septembre 2021.

Article L.111-1 du Code de la propriété intellectuelle. 


\title{
Espaces romanesques, espaces du souvenir : le chemin d'une écriture
}

\author{
Montserrat Mañes Serrano
}

1 Le titre du roman de Amin Maalouf, Léon l'Africain, suggère des attaches avec l'histoire que le contenu du récit ne fait que confirmer. Cependant, cette part indéniable de vérité se voit doublée d'une fiction qui transparait dans la division et les titres des livres et des chapitres, et qui s'épaissit de réalité en mouillant dans l'encre de l'écriture au fil des noms, des voyages, des lieux et des personnes que le narrateur nous montre. En tant que livre de voyages, l'espace y règne en maître ${ }^{1}$. En fait, il commande la création romanesque doublement: par son ancrage historique et par le choix narratif d'un personnage dynamique.

2 Maalouf a pris comme pivot central de la narration un personnage historique dont les écrits nous sont connus. Le reste est fiction: dans l'autobiographie fictive de Léon l'Africain nous découvrons les lieux visités et connus par lui, et ceux créés ou recréés par le scripteur. Les axes spatial et temporel sont indissolublement liés par l'organisation interne $d u$ récit: quarante chapitres, un pour chaque année du protagoniste, groupés en quatre livres, chacun portant le nom d'une ville: Grenade, Fès, Le Caire, Rome. Ce sont des indices clairs de l'importance de l'espace dans le déroulement du roman, et une confirmation de celui-ci comme livre de voyages, sinon comme le livre d'un voyageur'2.

Quand le récit commence, la boucle spatio-temporelle est fermée : Le voyageur est de retour; il raconte ses voyages à son fils, avant de se fixer et de se taire scripturalement, pour commencer un dernier trajet statique et intérieur : la descente silencieuse vers la mort et l'oubli. Devenu rapporteur d'espaces, Léon l'Africain nous permet de reparcourir, à travers ses souvenirs, les chemins et les bornes de sa vie errante. L'agencement de ces espaces et leur organisation romanesque doit nous permettre de découvrir la structure profonde du roman. Et, étant donné que le parcours narratif est linéaire, le point de départ de notre étude nous semble clair, d'autant plus que la doctrine du voyageur, annoncée dans la présentation, est reprise dans la clôture : les deux seules pages non datées spécifiquement, et appartenant au présent du narrateur. 
Selon ses propres mots, Je suis fils de la route, ma patrie est caravane, et ma vie la plus inattendue des traversées (9)3.

4 Le Livre de Grenade retrace le temps d'avant sa naissance et de son enfance, ce qui veut dire que le narrateur est spectateur non participant. Grenade, espace quasi mythique, appartient à la famille, au passé, aux ancêtres. Et il rapporte des descriptions, une topographie de la ville tirées des souvenirs familiaux : la mère, le père, l'oncle :

L'image qui s'offrait à ses yeux de fillette apeurée, ma mère ne l'oublierait jamais, pas plus que ne l'oublieraient tous ceux qui se trouvaient à Grenade en cette maudite journée de la Parade. Dans la vallée où coulait d'habitude le bruyant mais paisible Darro, voilà qu'un torrent démentiel s'était formé, balayant tout sur son passage, dévastant jardins et vergers, [...] jusqu'à former, en fin de journée, [...] une immense mare qui engloutissait la cour de la grande mosquée, la Césarée des négociants, le souk des bijoutiers et celui des forgerons (27).

5 La minutie descriptive est frappante, d'autant plus qu'il s'agit d'un espace que le narrateur n'a pas investi de sa présence ; il se peut que Maalouf veuille faire étalage de son savoir ; en tout cas, cette minutie, doublée de la confusion des voix narratives qui se produit souvent, donne une grande épaisseur de réalité à des souvenirs qui n'appartiennent pas en propre au narrateur. La ville de Grenade, perçue comme un lieu clos, englobe tous les autres lieux - ou presque - de ce premier livre, et présente une topographie spécifique aux espaces bien circonscrits. Ses alentours, décrits comme des vues d'ensemble ou parfois comme de simples descriptions topographiques, servent à ancrer la ville dans le vécu et dans la réalité géographique et historique connue. Nonobstant, l'espace textuel de ces descriptions ne coïncide pas forcément avec l'espace strictement référentiel (56) ${ }^{4}$. D'ailleurs, ces alentours ne sont pas visités par les rapporteurs-narrateurs, à quelques exceptions près, dont la raison semble être purement une référence historique : le séjour du père à Santa Fe notamment (78-80). Quant au port d'Almeria, il est chargé du point de vue spatial d'une valeur symbolique profonde : la mer apparait pour la première fois comme porte d'exil, lieu de liberté et d'évasion (108-110).

6 La maison, espace clos appartenant de droit à l'image de la femme et de la mère, est un topos privilégié; noyau spatial dans l'espace plus large de la ville, elle est un lieu sécurisant ${ }^{5}$ et cependant le moins décrit de tous. Il s'agit peut-être d'un refus de toucher au monde des femmes, chez le musulman Hassan ${ }^{6}$. Mais à l'intérieur de cette maison il est un lieu qui revient, habité par les hommes, regardé et visité par les femmes: le patio, le seul sous-espace qui a droit à quelques touches descriptives (19).

7 Le palais de l'Alhambra appartient au monde externe du social, de la politique. Perçu comme un lieu surélevé par rapport à la ville, lointain et fastueux, il est décrit partiellement par l'oncle - Ainsi, quand il me racontait la chute de Grenade, son récit débutait immanquablement dans les salles tapissées de l'Alhambra (64) -, avec de sommaires descriptions ambulatoires et statiques, où l'ensemble prime sur le détail :

Le premier jour de cette année-là j'étais monté de bonne heure à l'Alhambra, non pas pour joindre, comme à l'accoutumée, le petit bureau du diwan où je rédigeais les lettres du prince, mais pour présenter, avec quelques notables de ma famille, les vœux du Râs-es-Sana. Le majlis, la cour du sultan, qui se tenait pour l'occasion dans la salle des Ambassadeurs, grouillait de cadis enturbannés...(38).

A côté de ces sous-espaces fermés, les ruelles de l'Albaicin relient la maison au palais de l'Alhambra et à la ville, donc au monde externe. En tant que lieux de passage ces rues sont un espace externe par rapport aux antérieurs, et représentent le danger et le 
malheur face à la sécurité de la maison : les ruelles glissantes et périlleuses pour Warda et Salma enceintes (16) ; la rencontre avec le frère chrétien de l'esclave Warda, les révoltes, la guerre, la famine, l'insécurité... et surtout le « déluge » du jour de la Parade raconté par la mère (27); en ce point, le narrateur offre des aperçus descriptifs étonnamment détaillés (23-25) de la ville, l'Albaicin et même des intérieurs de maison (26) : le quartier de la Sabika, le jeu de la Tabla - les habitants de Grenade et des villages alentour avaient pris l'habitude de se rassembler, grands et petits, sur les pentes de la Sabika, au pied de l'Alhambra, près du cimetière, d'où ils pouvaient voir, au-dessus d'eux, l'interminable cérémonie (22-23). Le terrible orage de ce jour de la Parade favorise même l'inclusion d'une description à fonction mimésique de type voir? .

9 Le parcours des ruelles, les places, les événements rapportés au sein du labyrinthe mythique de la ville: des mémoires teintées de la couleur verte de l'Islam, qui doivent cependant beaucoup aux sources historiques connues, telles Las guerras Civiles de Granada de Pérez de $\mathrm{Hita}^{8}$ et autres. Mais la part de réalité et la part de fiction du Livre de Grenade importe peu dans ce cas-ci : ce qui est significatif du point de vue scriptural, est l'agencement romanesque de l'espace. Et il est intéressant de signaler comment le narrateur a soin de nommer fréquemment les lieux, dont quelques-uns reviennent souvent, et de marquer les traces des déplacements, souvent avec leurs bornes :

Elle passa devant la porte des Sept Etages, longea les remparts, sortit de la ville par

la porte de Nadj pour atteindre le Genil, dont la surface était gelée (79).

10 Il existe dans ce livre des lieux réservés aux différents personnages: le père qui transmet l'histoire générale de la ville et de la famille; Salma la mère pour raconter le menu détail, les souvenirs et les espaces qui ont marqué l'histoire et le devenir de la famille; l'oncle, possesseur des espaces et de la mémoire les plus séparés et inaccessibles: le palais, la politique, la connaissance du genre humain à travers les princes, et qui possède le pouvoir de faire revivre avec le son incantatoire de sa voix la magie de sa ville natale :...et sa voix était si rassurante qu'elle me faisait respirer les odeurs de ma Grenade natale, et sa prose était si envoûtante que mon chameau semblait avancer à son rythme (37). Mais il s'agit de lieux toujours circonscrits par des limites étroites: les murailles et les portes « infranchissables » de la ville, si ce n'est pour le départ définitif vers le premier exil.

11 Le Livre de Grenade se présente ainsi comme un espace limité, fermé, avec quelques sous-espaces liés entre eux par les rues de l'Albaicin, qui constituent dans la mémoire familière de Léon une patrie, un monde conservé sous un seul nom, devenu magique, statique et immuable : Grenade.

12 Le Livre de Fès, qui occupe une vingtaine d'années de Hassan, est de beaucoup le plus long'. Ce sont les années de son adolescence et de la force de l'âge. Le mouvement est essentiel dans sa structuration spatiale : l'espace est maintenant ouvert, parfois même éclaté. Les déplacements sont multiples : des voyages, des descriptions abondantes d'un monde exotique extérieur, mais aussi du monde fermé représenté par Fès, une ville qui offre au protagoniste la possibilité de l'apprentissage du déplacement et du départ.

La topographie spécifique privilégiée est d'abord l'espace fermé de la ville (115). L'invocation de Mohamed pour que l'enfant regarde ${ }^{10}$ inaugure une période descriptive à deux niveaux: partant d'une position fixe des descripteurs, nous avons une description du père à double fonction mathésique et sémiosique ${ }^{11}$. Le choix de ce qu'il donne à voir à son fils, avec des déictiques spatiaux typiques - là-bas, à gauche, à 
droite - a une fonction indicielle et un pouvoir cataphorique clair : il enchâsse dans ses mots le passé de la famille avec le futur de Hassan :

Là bas, dans cette plaine traversée par l'oued, c'est le cœur de la cité. A gauche, la rive des Andalous, fondée il y a des siècles par des émigrés de Cordoue; à droite, la rive des gens de Kairouan, avec, au milieu, la mosquée et l'école des Karaouiyines, ce vaste bâtiment aux tuiles vertes, où, si Dieu l'agrée, tu recevras l'enseignement des ulémas » (116)

La distribution spatiale par personnages se maintient avec quelques changements. Le destin brisé du père ne se refait pas : éternel exilé, il passe de l'espace fermé de Grenade à l'impossibilité de se fixer à l'intérieur de Fès. La répudiation de sa femme Salma et l'attachement à son passé représenté par Warda l'excluent définitivement de cette nouvelle patrie. Il reste hors la ville, cherchant à se fixer (160), et son espace demeurera multiple: Mohamed partageait son temps entre trois habitations, celles de ses femmes et sa maison de campagne, autour de laquelle ses cultures prospéraient (234). Reste l'espace investi par Hassan : étranger à la ville il connaîtra ses rues et ses endroits les plus cachés de la main d'un guide privilégié : Haroun le Furet (151).

Le noyau spatial de Fès-externe est le topo de la place, espace ouvert et lieu de passage par excellence qui se révèle un lieu privilégié de rencontres (128) et de découvertes pour les deux adolescents : leur Place des Prodiges est le pivot sur lequel tourne la ville et de laquelle tous les chemins partent :

La Place des Prodiges occupait le croisement de plusieurs rues passantes. L'une, encombrée par les libraires et les écrivains publics, débouchait sur le parvis de la Grande-Mosquée ; une autre abritait les vendeurs de brodequins et de souliers ; une troisième les marchands de brides, de selles et d'étriers; la quatrième enfin était pour nous un passage obligé. Là se trouvaient les laitiers,... (150-151).

Léon inscrit dans le système spatial de ce livre des parenthèses dont la géographie échappe à la topographie dans laquelle se meuvent les personnages; des espaces tels que le Rif, qu'il décrit avec des souvenirs postérieurs à la temporalité du récit (177); la prise de Mélilla par les castillans (146), ou la parenthèse dédiée à l'espace lointain de Grenade (154-158).

17 Ce sont des années de caravane, ce qui veut dire que même dans l'éclatement spatial que suppose ce périple avec ses étapes, le noyau de la ville comme entité fermée ou pseudo-fermée continue d'exister: pour Léon, une caravane est en fait une ville ambulante : Vue de loin, la caravane est un cortège; vue de près, c'est un village [...] pour lequel toutes les contrées sont lointaines, même celle d'où l'on vient, même celles qu'on traverse (207). Nous retrouvons des descriptions de villes, de paysages, de coutumes, et un espace géographique privilégié : Tombouctou, éden au bout du désert, ville de la découverte de l'amour située sans erreur possible (217). Ses espaces intérieurs, très peu singularisés $(223,225)$, prévalent sur les extérieurs, mais l'attirance de la ville externe (226) devient évidente par sa première vision sur ses rues ${ }^{12}$.

Le chapitre de L'année de fortune rapporte son deuxième grand voyage, celui de la richesse matérielle et de la renommée politique; les marques spatiales ne sont pas abondantes. L'intérêt réside exclusivement ici dans le fait même du voyage comme moyen et instrument de changement moral et social, car Hassan rentre changé à Fès ${ }^{13}$. Et son départ pour l'exil, après des années de richesse et d'honneurs (282), est soigneusement décrit, ainsi que son parcours en compagnie de Hiba. L'abondance des jalons géographiques et temporels soulignent son importance : 
Quatre jours plus tard, nous traversions le col des Corbeaux, par un temps sensiblement plus froid que je ne l'aurais supposé en ce mois d'octobre. Quand il fallut s'arrêter pour la nuit, les gardes installèrent le campement dans une petite dépression entre deux collines [...] Ils formèrent un cercle grossier de tentes, au milieu duquel s'élevait la mienne, véritable palais de toile aux pans ornés de versets coraniques artistement calligraphiés (283).

19 En fait, il marque un tournant dans la vie de Hassan : il perdra sa fortune et Hiba, mais celle-ci, devenue image du destin et de la vie (285), lui permettra une deuxième naissance symbolisée spatialement par ce quasi ventre maternel qu'est le refuge de la caverne: un gîte exigu, plutôt une cavité dans le roc qu'une véritable galerie (284), cocon maternel qui ne s'est ouvert qu'au troisième jour.

20 Du point de vue de la spatialité, la ville, l'un des topoi autour duquel s'organise la spatialité du roman, est dans ce livre le point d'attache et le port duquel on part et auquel on retourne : c'est ainsi que son premier retour à Fès suppose un arrêt temporel et spatial, la sédentarisation de Hassan qui agit en homme d'affaires, en homme politique et en mari. Et Fatima, sa première épouse, sera mal aimée et peu attrayante, comme la vie immobile l'est pour lui. Quant à la maison, elle acquiert une dimension symbolique différente; car Hassan, devenu riche, a beau se passionner pour la construction de sa maison $(264,270)$, celle-ci demeurera toujours en chantier, signe de l'impossibilité pour le héros de se fixer (330-331).

21 Le Livre de Fès, ce noyau spatial multiple et multiplié, commençait par le voyage de Grenade à Almeria, et l'arrivée au Maghreb ; il se termine par une traversée étonnante à travers l'Afrique vers l'Égypte :

...je connais le nom de soixante royaumes dont quinze que j'ai traversés l'un après l'autre cette année-là, du Niger au Nil.[...] je n'ai fait que suivre la route habituelle des caravanes qui partent de Djenné, du Mali, d'Oualata ou de Tombouctou vers Le Caire. [...] Il ne nous fallut pas plus de douze jours pour atteindre, en longeant le Niger, la ville de Gao. Elle n'avait pas de mur d'enceinte, mais aucun ennemi n'osait s'en approcher,[...]. Les étapes suivantes nous firent traverser plusieurs royaumes, parmi lesquels je mentionnerai ceux d'Ouangara, de Zegzeg, de Cano, bien plus important que les précédents, mais où nous évitâmes de nous attarder ... (292).

Ces espaces, traversés sans aucun souci descriptif, ne sont que des lieux de passage qui transmettent l'impression d'une spatialité éclatée. Et si au début du livre la mer était le milieu qui exprimait directement un bouleversement profond dans la vie du héros, ici l'équivalent est le voyage du Nil : l'élément liquide s'avère être un motif descriptif qui symbolise le passage, le devenir.

23 Le Livre du Caire donne l'impression d'un rythme vertigineux : les voyages se succèdent sans répit, dans une continuelle accélération. Il transmet aussi l'idée de circularité, car Hassan revient parfois sur ses pas, et certains endroits se répètent. Fès devient un point d'inflexion : sans possibilité aucune d'y refaire sa vie, il quitte pour toujours un espace où il s'est senti étranger de tout temps, et son errance recommence : Je ne possédais plus à Fès qu'un chantier, une ruine peuplée de regrets et vide de souvenirs (335). Cependant, la distribution spatiale est quasiment la même que celle que l'on retrouve dans le Livre de Fès; en outre, nous voyons réapparaître les mêmes topoi et les mêmes motifs privilégiés utilisés dans les deux livres antérieurs : la place, la porte, la maison, la ville.

24 Le système spatial de la ville du Caire est construit autour de deux pôles au développement inégal : le Caire, espace urbain, et la parenthèse du village près des Pyramides, espace rural avec comme sous-espace la maison de ses rencontres 
amoureuses avec la Circassienne. Les lieux sont nommés : rue Saliba, Bab Zuwaila, porte du départ et de la fuite comme celle de Najd à Grenade, la Place de l'Ezbékieh, lieu de rencontres et de rassemblements comme la Place des Prodiges :

La Place de l'Ezbékieh s'emplissait lentement à mesure que les mosquées se vidaient, car c'était l'habitude de tous les Cairotes de s'y rassembler après la cérémonie pour jouer aux dés, écouter les boniments des conteurs, se perdre parfois en fin d'après-midi dans les ruelles avoisinantes où certaines tavernes proposaient un raccourci pour l'Éden (318).

En investissant la ville (311) il en prend possession par ses randonnées; l'effet réaliste de ces promenades est clair, car il ne se retient pas de nommer et de nous amener avec lui à travers les rues et les quartiers $(307,309,311,314 . .$.$) . Nous avons donc la ville$ comme espace fermé et externe par rapport à la maison, qui est ici plus qu'ailleurs un point d'ancrage sécurisant: située topographiquement et décrite par personne interposée (302), la maison l'attire et lui appartient dès le début (306).

Entraîné par Nour, encore une femme qui le pousse au voyage, il recommence son périple, avec des départs constants par mer, de brefs séjours, des étapes privilégiées comme La

Mecque ou Tunis, où il a l'espoir de jeter l'ancre [...] pour quelques années au moins (340), des villes qui se succèdent et se répètent, des rencontres et des batailles $(353,358,363 .$.$) ,$ jusqu'à sa dernière étape dans le port de l'île de Djerba, d'où il partira, une fois de plus en bateau, pour une nouvelle vie. Et c'est de la bouche de Nour que sortent les mots révélateurs qui poussent Hassan à montrer quelle est sa conception de la vie :

« De quelle pâte es-tu fait pour accepter de perdre une ville après l'autre, une patrie après l'autre, une femme après l'autre, sans jamais te battre, sans jamais regretter, sans jamais te retourner?

- Entre l'Andalousie que j'ai quittée et le Paradis qui m'est promis, la vie n'est qu'une traversée. Je ne vais nulle part, je ne convoite rien, je ne m'accroche à rien, je fais confiance à ma passion de vivre, à mon instinct du bonheur, ainsi qu'à la Providence. » (348)

Celui qui se laisse emporter par le courant de la vie, celui qui est toujours entre deux étapes d'un voyage - Les bruits du lointain m'appelaient, il était écrit que je ne resterais pas sourd à leurs tentations (364) - commencera alors son dernier et long voyage vers un autre exil : le Livre de Rome débute dans le ventre d'un bateau (379), et cette presque absence de spatialisation au fond de la cale devient symboliquement l'attente d'une nouvelle naissance. Celle-ci comporte un nouveau nom, ce qui revient à dire une nouvelle identité, et de nouveaux savoirs à acquérir, au beau milieu d'une civilisation inconnue pour lui : l'Europe de la Renaissance, la cour papale, Rome...

L'organisation spatiale de ce dernier livre évoque une idée de spatialité concentrée, à tel point que nous devrions peut-être parler, au lieu de degrés d'ouverture, de degrés de fermeture. Le noyau est la ville de Rome, espace limité et aux lieux concentriques : Hassan-Léon, arrivé esclave de Naples à Rome, est enfermé puis logé dans un cachot du Château Saint-Ange :

Je fus installé dans une petite pièce, meublée d'un lit, d'une chaise et d'un coffre en bois, comme s'il s'agissait d'une modeste hôtellerie plutôt que d'une prison, exception faite de la lourde porte, dûment cadenassée de l'extérieur. (382)

Dans une deuxième étape, son espace se dilate jusqu'à occuper le château (387); un dernier élargissement spatial lui permet de visiter la ville (393). A la fin de son séjour le chemin s'inverse: homme libre, il est emprisonné dans la même cellule qu'il avait 
occupée jadis; lors du sac de Rome, le château devient son refuge : il occupe avec sa famille son ancienne chambre comme lieu de résidence ${ }^{14}(461)$.

Pour ce qui est des espaces atteignant la ville, celle-ci nous est offerte, en tant que lieu extérieur, sur ses deux visages: accompagné de Guicciardini, il visitera la Rome monumentale. Lors de sa promenade avec le luthérien Hans, et suivant une topographie bien réaliste des lieux que les déictiques renforcent, nous retrouvons la ville de la pauvreté et de la misère humaine :

Je me dirigeai d'abord tout droit, vers la rue des Vieilles-Banques, avant de m'engager, à gauche, dans la célèbre rue del Pellegrino, afin d'y admirer les devantures des orfèvres et les étalages des marchands de soie.[...]Il m'entraîna par des ruelles étroites, où il était impossible de passer sans enjamber des amas d'immondices. Puis, dans le lieu le plus sombre, le plus puant, il s'arrêta net (393-394).

Étant donné que les sorties de la ville sont rares, les lieux ouverts sont peu abondants. Il existe des lieux externes rapportés : ceux de Maddalena, si semblables aux siens ; ou des espaces rapportés qui sont ceux du pouvoir et de la politique (428-429), des guerres et des batailles $(421,436)$. Les visites à d'autres espaces et à d'autres villes, certes, existent. Parmi celles-ci, Florence (451-452) prend une ampleur intéressante du point de vue de l'étude spatiale ${ }^{15}$, car Léon nous offre une description ambulatoire qui ressemble fort à une visite guidée ; la rue, la cour du palais des Médicis, le Dôme, la piazza della Signoria, le Ponte Vecchio...

33 A l'espace qui se rétrécit dans le Livre de Rome il faut ajouter la superposition temporelle du passé : les faits se succèdent comme un écho lointain. La tempête de neige (436) lors de son retour de Pavie, et dans laquelle il a failli mourir, est aussi dangereuse que celle de l'Atlas, où Hiba lui sauva la vie. L'espoir des habitants de Rome assiégée lui rappelle trop les derniers jours de Grenade (457); la vision de la ville envahie (463) fait penser à Tombouctou en flammes; et le souvenir lointain du jour de la Parade à Grenade, raconté par sa mère, se superpose aux événements romains du jour signalé pour conclure l'accord de paix :

Je me trouvais au milieu de la foule, place Saint Pierre, pour assister à ce moment de délivrance. Il faisait beau, une superbe journée de printemps, quand le dignitaire apparût, entouré de sa garde. Mais, à l'instant où il franchit la porte du Vatican, il y eut un éclair, suivi d'une pluie diluvienne qui s'abattit sur nous avec un vacarme de fin du monde. La surprise passée, je courus m'abriter sous un porche, bientôt assiégé par une mer de boue (459-460).

Le nouvel exil, vécu comme une délivrance, s'avérera une décalque des antérieurs et commencera à Naples, sur le même port où débutait ce Livre de Rome. Le motif spatial de la porte est moins utilisé, et la sortie de Rome est une succession d'étapes dont les portes sont les sauf-conduits avec le nom de divers chefs militaires. La dernière, ouverte vers la liberté et vers la mer, si elle existe, porte le nom d'un homme, et a la figure d'un bateau: La plus belle des galées d'Abbad nous attendait, prête à cingler vers Tunis (471). Le livre de la vie de Léon l'Africain se ferme aux approches de la côte africaine par une exhortation à son fils, qu'il appelle à tous les voyages possibles: N'hésite jamais à t'éloigner, au-delà de toutes les mers, au-delà de toutes les frontières, de toutes les patries, de toutes les croyances (473).

Nous voyons comment les mêmes topoi reviennent de livre en livre, de déplacement en déplacement, d'exil en exil. Mais il est un motif qui les relie tous d'une certaine façon : l'image de la femme. Ancrée dans la spatialité éclatée du livre pris comme entité 
unique, elle est inséparable de l'idée de voyage. Et on peut observer comment chaque ville est liée à l'existence d'une femme : sa mère pour Grenade : symboliquement, l'exil est déjà ici lié à la femme ; l'exil de l'enfant par rapport à la mère, à l'espace utérin, et l'exil de l'homme par rapport à son pays. Fès est douée d'une double spatialité : en tant que ville de l'immobilité absolue, elle est liée à Fatima, sa première femme, négation de l'amour et de la beauté. En tant que porte et point de départ, Fès lui ouvre le désert, le voyage et le « havre » de Tombouctou, ville de la découverte de l'amour, et le territoire de Hiba, la beauté. Le Caire est la paix, mais aussi l'intranquillité de la Circassienne, le bizarre et l'aventure au cœur du réel.

Rome ferme la boucle, cette ronde des villes : la dernière femme a ses mêmes racines, et elle est doublement exilée: Grenadine comme lui, et de surcroît juive, donc appartenant au peuple de l'exil par excellence. Maddalena-Judith porte comme lui un double nom et une double identité. C'est ainsi que dans Rome et dans cette femme, images toutes les deux de permanence et du passage, se retrouvent et se joignent toutes les routes, que tous les espaces convergent vers le repos définitif: espace hors du temps, et secret dorénavant pour le lecteur.

\section{NOTES}

1. Cf. BouRneuf, R., OUeLLET, R : L'univers du roman, Paris, P.U.F., 1972, (1985).

2. Le voyage, de par la nature même du roman, est un thème privilégié. Comme le souligne LE CALVEZ, E.,» La structure de l'espace romanesque est le produit d'une structuration de thèmes »: «Structurer le topos et sa graphie », in Poétique, 78, Seuil 1989, p. 156.

3. Les indication des pages renvoient à Amin MAALOUF, Léon l'Africain, Paris, J.-C. Lattès, 1986.

4. Cf. à ce propos Goldenstein, J.-P. : Pour lire le roman, Paris, Deboeck-Duculot, 1986, p. 89.

5. Cf. ReUTeR, Y.:Introduction à l'analyse du roman, Paris, Bordas, 1991, pp. 54-56. Cf. aussi BACHELARD, G. : La poétique de l'espace, Paris, P.U.F. 1981 (1957).

6. Mais la réalité externe pénètre dans ce lieu fermé à travers les hommes - le père, l'oncle -, et surtout grâce à un personnage absolument libre: la juive Sarah la Bariolée, pour qui les frontières, les espaces interdits, n'existent pas. Joker qui court de rue en rue, de maison en maison, qui traverse les mers et possède les villes, qui connaît les secrets des cœurs et les racontars du peuple, et qui sait, grâce à l'expérience séculaire de son peuple, les réalités passées, présentes et futures.

7. Salma enfant justifie l'existence de cette description par sa seule présence dans un lieu privilégié duquel elle domine la ville rasée. Cf. Adam, J/-M., Petitjean, A. : Le texte descriptif, Nathan : Paris 1989, pp. 37-42.

8. La vision historique est double : dans le roman, Mohamed fait partie des cinq cents notables amenés en otages au roi Ferdinand (78) ; dans l'histoire des Guerras Civiles de Granada de Pérez de Hita, ces notables se rendent à Santa Fe de leur plein gré : Acordado esto desta manera, començaron de tratar entre ellos los que avían de yr a hablar al Rey Don Fernando sobre ello, y al fin los que fueron a tratarlo eran los Alabezes, $y$ Aldoradines, $y$ Gazules, $y$ Vanegas, $y$ Muça por cabeça de todos ellos ; [...]El qual [le roi Ferdinand], como viesse venir tan grande esquadrón, mandó que todo el Real se apercibiesse por si fuesse menester... (PeREz De HITA, G. : Guerras Civiles de Granada, $1^{\mathrm{e}}$ parte, Madrid, E. Bailly-Baillière, 
1913, p. 287). Cependant, à la lecture parallèle des deux textes, un fait reste invariable : la liberté dont ils jouissent à l'intérieur de Santa Fe.

9. Dans le Livre de Fès se trouve l'ancrage avec la réalité le plus clair de la vie du vrai Léon l'Africain : son voyage d'Afrique, trois ans qui représentent dans le roman l'entrée dans l'âge adulte, la rencontre avec l'amour, l'ouverture totale au monde extérieur.

10. Le rôle de ces deux personnages, en épousant la thématique du regard, est exclusivement celui de descripteurs. Cf. Milly, J. : Poétique des textes, Paris, Nathan Université, 1992, p. 142.

11. Toujours d'après la nomenclature de ADAM, J.-M., PeTITJEAN, A.: Op. cit., pp. 26-33.

12. L'ancrage temporel (223) introduit une description classique du type voir, avec des verbes de perception et un milieu transparent qui offre la possibilité d'observer $\mathrm{Cf}$. HAMON, Ph., Introduction à l'analyse du descriptif, Paris, Hachette, 1981, pp. 186-189, et J. M. ADAM, J.-M., PETITJEAN, A., Op. cit. p. 40, dans la typologie qui découle de l'insertion de la description dans le récit.

13. Y. REUTER remarque comment les lieux non seulement peuvent « symboliser tel statut ou tel désir ", mais "signifient aussi des étapes de la vie, l'ascension ou la dégradation sociale, des racines ou des souvenirs » (Op. cit. pp. 55-56).

14. La maison reste le lieu sécurisant par excellence, et nous trouvons dans ce dernier livre la seule description minutieuse de ce type d'espace fermé : sa situation topographique - le quartier du Ponte - et sa disposition intérieure (410-411).

15. Ce type de descriptions renvoie plus ou moins directement à la tradition rhétorique, qui demande dans toute description une qualité première : l'evidentia. De plus, comme J. Molino le fait remarquer, «Décrire, c'est faire voir et faire croire, c'est faire croire qu'on voit, qu'on sent, qu'on touche, et qu'on aurait envie, comme les oiseaux, de se poser sur le tableau où Zeuxis avait peint une grappe de raisin ». In « Logiques de la description », Poétique, 91, Seuil 1992, p. 378.

\section{AUTEUR}

\section{MONTSERRAT MAÑES SERRANO}

Université de Grenade 